\title{
„Hirn-Doping“: Erythropoietin zur Verbesserung kognitiver Leistung bei Mensch und Maus
}

Einleitung: Kognitive Leistung ist bei vielen neuropsychiatrischen Störungen beeinträchtigt, aber effektive Therapien fehlen. Im Rahmen klinischer Studien mit Patientengruppen mit Krankheiten so divers wie chronische Schizophrenie, chronischprogrediente Multiple Sklerose, behandlungsresistente schwere Depression, aber auch bipolare Erkrankung, fanden wir über die letzten 15 Jahre hinweg immer wieder, dass rekombinantes humanes Erythropoietin (EPO) zuverlässig zu einer anhaltenden Verbesserung höherer kognitiver Funktionen führt. Insbesondere Exekutivleistungen, Lernen, Gedächtnis, Aufmerksamkeit und Geschwindigkeit der Informations-Verarbeitung sowie zusätzlich Motivation und Antrieb waren bei allen untersuchten Krankheitsbildern unter EPO verbessert. Zudem fand sich eine Verminderung des Verlustes an grauer Substanz durch EPO in zwei unabhängigen Studien, bei Schizophrenie und bei affektiven $\mathrm{Er}$ krankungen. Interessanterweise co-determiniert selbst normale genetische Variation in den Genen, welche EPO und EPORezeptor kodieren, unsere kognitive Leistungsfähigkeit. Insgesamt finden unsere Patientenstudien mit Doping als „optimaler Feldstudie zur EPO-Wirksamkeit“ überzeugende Unterstützung: Auch wenn Doping im Sport abzulehnen ist, sollte doch „Hirn-Doping“ für die Behandlung von Patienten mit neuropsychiatrischen Erkrankungen erwogen werden.

Gegenwärtige Situation der EPO-Anwendung bei neuropsychiatrischen Indikationen: Leider stagniert - insbesondere aufgrund der längst ausgelaufenen EPO-Patente - die klinische Entwicklung von EPO für die Indikation Hirnerkrankungen. Trotz der vielversprechenden Ergebnisse und des Fehlens anderer - auch nur halbwegs EPO an Effektivität gleichkommender Substanzen, gelang es bislang nicht, Geldgeber für die notwendigen klinischen Phase III-Studien zu finden. Deshalb fokussieren wir unsere EPO-Forschung gegenwärtig auf Tiermodelle, die es uns ermöglichen, umfassende mechanistische Erklärungen für die hohe Wirksamkeit von EPO auf Kognition und Plastizität, Neuroprotektion und Neuroregeneration zu erhalten. Darüber hinaus beschäftigen wir uns mit den hierbei involvierten EPO-Rezeptoren (EPOR). Wir erforschen also die (Patho)Physiologie des EPO/EPOR-Systems im Gehirn.

Methoden: Um den Effekt von EPO im Gehirn zu studieren, setzen wir gesunde Mäuse und viele verschiedene genetische Mausmodelle ein (z.B. konditionale EPOoder EPOR-KO-Mäuse oder NG2-CreERT2: R26R-td-tomato-mEGFP, Gli-Cre-, NestinCre-, Sox2-Cre-, CX3CR1-Cre-Linien zum „Fate Tracking“), in welchen u.a. höhere kognitive Leistung, MRI-Volumetrie und hippocampale Elektrophysiologie getestet werden. Zusätzlich erheben wir umfangreiche Daten an Hirnschnitten und in der Zellkultur, basierend auf Immunhistochemie, qPCR, Western Blot, in situ-Hybridisierung, konfokaler Mikroskopie und NanoSIMS (nanoscopic secondary ion-massspectrometry) Technologie. Darüber hinaus arbeiten wir mit verschiedenen Zellkultursystemen (neuronalen Stammzellen, Neurosphären und E17 Hippocampus-Neuronen von Mäusen oder humanen IPSC-derivierte Neuronen).

Ergebnisse und Diskussion: Wir fanden, dass EPO auch bei gesunden Mäusen zu einer markanten Verbesserung kognitiver Leistung führt, vergesellschaftet mit einer Steigerung der hippocampalen Langzeitpotenzierung. Auf zellulärer Ebene entdeckten wir nach einer 3-wöchigen EPOBehandlung eine Vermehrung der Zahl von Pyramidenneuronen und Oligodendrozyten im Hippocampus um erstaunliche $20 \%$. Diese dramatische Erhöhung funktionell integrierter Zellen erfolgte ohne vermehrte Proliferation und ohne Apoptose-Reduktion. EPO führt, wie wir in der Folge feststellen konnten, zu einer raschen Differenzierung lokaler Vorläuferzellen zu Neuronen und Oligodendrozyten. Mit diesen Befunden spürten wir völlig unerwartet neue Mechanismen postnataler Neurogenese, bedarfsadaptierter Plastizität und Neuroregeneration auf. Obwohl der Beweis beim Menschen für genau dieselben Mechanismen noch aussteht, dürften die EPO-Effekte auf Hirnsubstanzminderung, welche im MRT nachweisbar waren, in diese Richtung weisen.

Ausblick: Ein Schwerpunkt unserer EPOForschungsarbeit richtet sich derzeit auf die Induktion von endogenem EPO im Gehirn durch physiologische Hypoxie. Mit unseren laufenden Experimenten zur erstmaligen Isolierung und biochemischen Charakterisierung der EPO-Rezeptoren im menschlichen Gehirn, welche sich zumindest teilweise vom „klassischen“ hämatopoietischen EPO-Rezeptor unterscheiden, erhoffen wir uns nicht nur ein besseres Verständnis der (Patho)Physiologie des EPO-Systems im Gehirn, sondern möglicherweise auch völlig neue Behandlungsansätze.

\section{Interessenkonflikte}

Patente für den Einsatz von EPO bei Schlaganfall, Schizophrenie und Multipler Sklerose („usage Patente“).

\section{Autor}

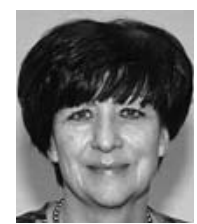

Hannelore Ehrenreich Klinische Neurowissenschaften, Max-Planck-Institut für Experimentelle Medizin, und DFG Forschungszentrum CNMPB, Göttingen

Korrespondenzadresse

Prof. Dr. Dr. Hannelore Ehrenreich Klinische Neurowissenschaften Max-Planck-Institut für Experimentelle Medizin, und DFG Forschungszentrum CNMPB Hermann-Rein-Straße 3

37075 Göttingen

ehrenreich@em.mpg.de

Literatur

[1] Hassouna I, Ott C, Dahm L et al. Revisiting adult neurogenesis and the role of erythropoietin for neuronal and oligodendroglial differentiation in the hippocampus. Mol Psychiatry 2016, doi: $10.1038 / \mathrm{mp} .2015 .212$ 
[2] Wüstenberg T, Begemann M, Bartels C et al. Recombinant human erythropoietin delays loss of gray matter in chronic schizophrenia. Mol Psychiatry 2011; 16: 26-36

[3] El-Kordi A, Radyushkin K, Ehrenreich $\mathrm{H}$. Erythropoietin improves operant conditioning and stability of cognitive performance in mice. BMC Biol 2009; 7: 37
[4] Adamcio B, Sargin D, Stradomska A et al. Erythropoietin enhances hippocampal longterm potentiation and memory. BMC Biol 2008; 6: 37

[5] Ehrenreich H, Fischer B, Norra C et al. Exploring recombinant human erythropoietin in chronic progressive multiple sclerosis. Brain 2007; 130: 2577-2588
[6] Ehrenreich H, Hinze-Selch D, Stawicki S et al. Improvement of cognitive functions in chronic schizophrenic patients by recombinant human erythropoietin. Mol Psychiatry 2007; 12: 206-220 